\title{
Infodemic, Misinformation and Disinformation in Pandemics: Scientific Landscape and the Road Ahead for Public Health Informatics Research
}

\author{
Javad POOL ${ }^{\mathrm{a}, 1}$, Farhad FATEHI ${ }^{\mathrm{b}, \mathrm{a}}$ and Saeed AKHLAGHPOUR ${ }^{\mathrm{a}}$

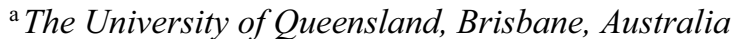 \\ ${ }^{\mathrm{b}}$ Monash University, Melbourne, Australia
}

\begin{abstract}
In response to epidemics and pandemics, access to authentic sources of information plays a critical role in informing public health practices. However, infodemic, i.e., an overabundance of health information, misinformation, and disinformation, impede implementing best public health policies during a public health crisis such as COVID-19. In this bibliometric study, we aim to report on concept mapping of infodemic literature, and in line with the World Health Organization (WHO)'s repeated calls for actions in managing infodemic, we highlight fruitful avenues for future directions. Through a visualization approach on a set of 414 records, a concept mapping was carried out. This map revealed 42 infodemic-related nodes in five clusters. We also propose an infodemic research platform in which a combination of the research nodes (e.g., COVID, pandemic, disinformation, fake news, post-truth, fact-checking, social networks, Facebook, WhatsApp, and lockdown) with impactful questions suggest future directions.
\end{abstract}

Keywords. Health information systems, public health informatics, infodemic, epidemic, pandemic, COVID-19, misinformation, disinformation, social media

\section{Introduction}

During a public health crisis such as the COVID-19 pandemic, media (online and offline) develop and disseminate useful information to inform decisions. Utilizing digital platforms, especially social media, is an effective strategy for achieving high spreads of public health content $[1,2]$. However, technology can be misused for producing and distributing misinformation (false or inaccurate information, regardless of intention) and disinformation (deliberately misleading information). On the dark side, in the online environment, false news diffuses significantly faster, and deeper than trustworthy information [3]. The spread of misinformation and disinformation during epidemics and pandemics poses a critical challenge to the public health, causing public panic and eroding public trust $[4,5]$.

Infodemic, a newly emerging word in the English vocabulary, is defined as "an overabundance of information and the rapid spread of misleading or fabricated news,

${ }^{1}$ Corresponding Author: Javad Pool, The University of Queensland, Brisbane, Australia; E-mail: j.pool@uq.net.au. 
images, and videos" [5]. Infodemic activities are "deliberate attempts to disseminate wrong information to undermine the public health response" [6]. The main digital environment that can be misused and may amplify infodemic is social media such as Twitter, Instagram, and Facebook. For example, a study on a sample of COVID-19 misinformation has revealed social media platforms as the main source $(88 \%)$ of infodemic.

Living in the world of COVID-19 information, misinformation, and disinformation, the World Health Organization (WHO) is emphasizing on flattening the "infodemic curve" [7]. In collaboration with digital platforms, including Google, TikTok, WhatsApp, and YouTube, WHO encourages consuming COVID-19 information from official and scientific sources [5]. It further calls on technologists and researchers for designing effective strategies and methods against misinformation and disinformation [6]. Although the infodemic topic has recently received attention among scholars, our understanding of the current status of infodemic-related research is limited. Inspired by WHO calls for responding to the pandemic misinformation and disinformation, we aimed to facilitate contributions to infodemic discourse and literature. Through this research, we report on the current infodemic research landscape and propose a platform for future directions in the digital sphere.

\section{Method}

To undertake a concept mapping of COVID-19 infodemics, we searched in the Web of Science database using topics related to infodemic, misinformation, and disinformation in January 2021. Our search strategy is shown in Table 1.

Table 1. Search strategy

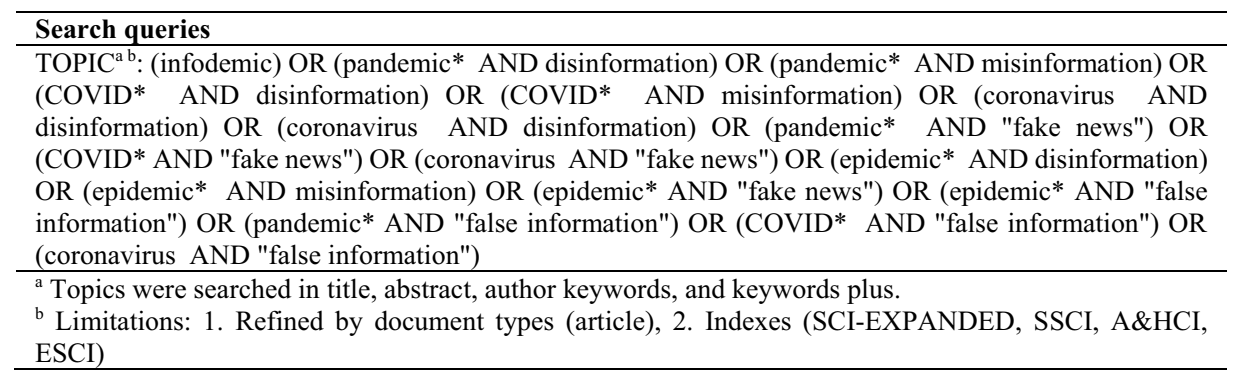

After the identification of records, we exported the results in a Tab-delimited format. Concept mapping of literature was conducted via VOSviewer software by importing the Web of Science file [8]. Type, unit of analysis, and counting method were co-occurrence, author keywords, and full counting, respectively. We, further, created a VOSviewer thesaurus file to remove or replace keywords. For example, COVID-19, COVID-19 pandemic, coronavirus, Coronavirus infections, SARS-CoV-2, Coronavirus disease 2019 were replaced by COVID. 


\section{Results}

414 records, published between 1993 and 2021, were identified by searching WoS database. Nearly 84 percent of records are published in 2020 . We divided our results into two subsequent sections: 'research clusters and concept mapping', and 'future directions'. To illustrate a concept mapping of infodemic literature and identify research clusters, 'network visualization' was applied. Based on the infodemic concepts, we also proposed important research questions and directions for future research.

\subsection{Research clusters and concept mapping}

An analysis of the co-occurrence of authors' keywords in 414 articles revealed five infodemic clusters and 42 research nodes, which are depicted in Figure 1.

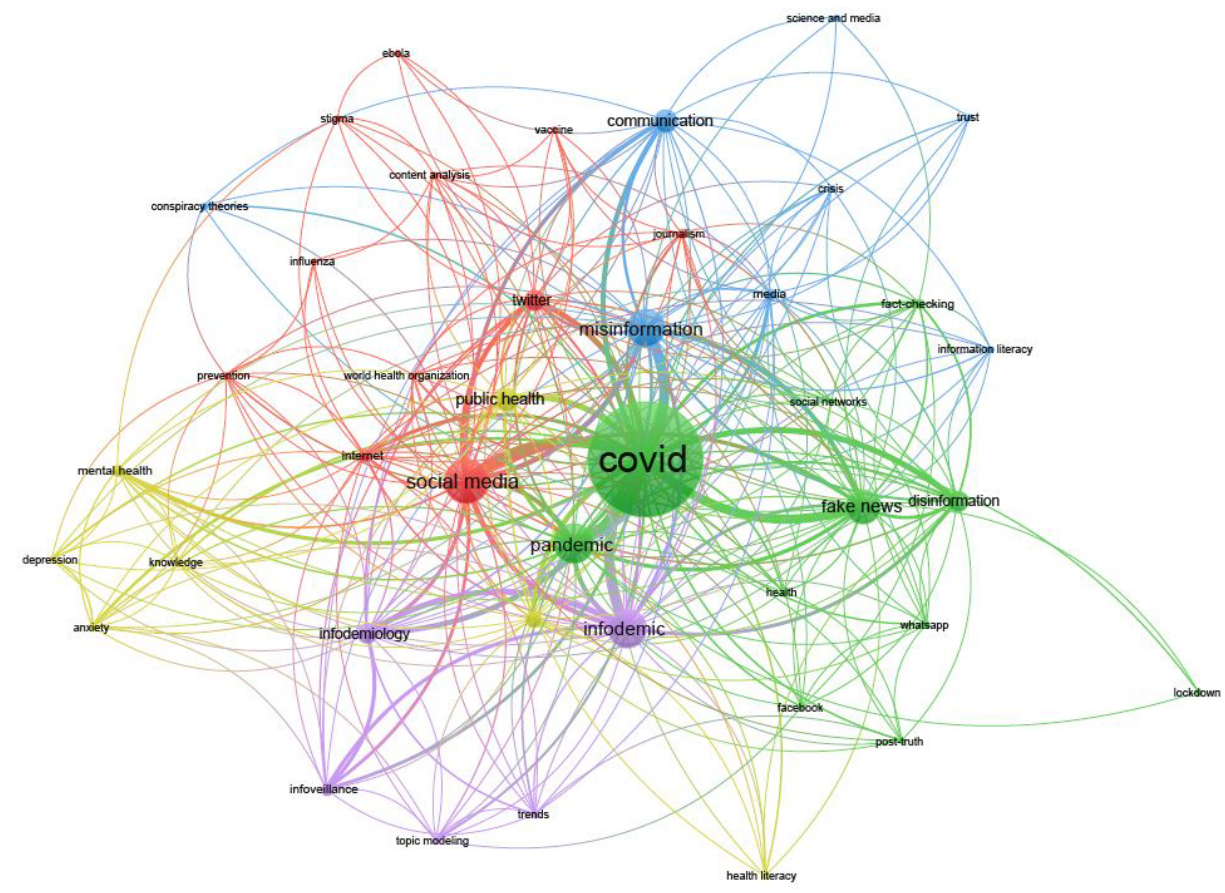

Figure 1. Network visualization of infodemic literature.

These clusters labeled as five research concepts: 'infodemic fabrication context and evaluation', 'infodemic digital tools and agency of actors', 'infodemic crisis management', 'infodemic knowledge and mental health impact', and 'studying and modeling the infodemic trends and topics'. Table 2 reports the research concepts and nodes in infodemic.

As evident in the visualization, it is not surprising that the COVID topic has received the highest attention among infodemic nodes. Other nodes including social media, misinformation, fake news, and pandemic are also important infodemic-related topics on the map. 
Table 2. Infodemic research concepts and hot topics across 414 identified records published in 1993-2021

\begin{tabular}{|c|c|c|}
\hline Cluster & Concept & Nodes $(n=42)$ \\
\hline $\begin{array}{l}\# 1 \\
\text { (Green) }\end{array}$ & $\begin{array}{l}\text { Infodemic fabrication context } \\
\text { and evaluation }\end{array}$ & $\begin{array}{l}\text { COVID, pandemic, health, disinformation, fake news, post- } \\
\text { truth, fact-checking, social networks, Facebook, WhatsApp, } \\
\text { lockdown }(n=11)\end{array}$ \\
\hline $\begin{array}{l}\# 2 \\
(\text { Red) }\end{array}$ & $\begin{array}{l}\text { Infodemic digital tools and } \\
\text { agency of actors }\end{array}$ & $\begin{array}{l}\text { Internet, social media, Twitter, journalism, content analysis, } \\
\text { prevention, influenza, Ebola, stigma, vaccine, World Health } \\
\text { Organization }(n=11)\end{array}$ \\
\hline $\begin{array}{l}\# 3 \\
\text { (Blue) }\end{array}$ & Infodemic crisis management & $\begin{array}{l}\text { Information literacy, science and media, communication, } \\
\text { conspiracy theory, crisis, media, misinformation, trust }(n=8)\end{array}$ \\
\hline $\begin{array}{l}\# 4 \\
(\text { Gold })\end{array}$ & $\begin{array}{l}\text { Infodemic knowledge and } \\
\text { mental health impact }\end{array}$ & $\begin{array}{l}\text { Anxiety, depression, mental health, public health, health } \\
\text { information, knowledge, health literacy }(n=7)\end{array}$ \\
\hline $\begin{array}{l}\# 5 \\
\text { (Purple) }\end{array}$ & $\begin{array}{l}\text { Studying and modeling the } \\
\text { infodemic trends and topics }\end{array}$ & $\begin{array}{l}\text { Infodemic, infodemiology, infoveillance, trends, topic } \\
\text { modeling }(\mathrm{n}=5)\end{array}$ \\
\hline
\end{tabular}

\subsection{Future directions}

Building upon the research concepts and integration of infodemic-related nodes (Figure 1), our illustrative directions for future research are summarized in Table 3.

Table 3. Future research direction in infodemic

\section{Concepts}

Infodemic fabrication context and evaluation
Why and how are COVID-19 infodemics (disinformation, fake news) in social media (e.g., Twitter, Facebook, WhatsApp) produced and consumed at national and global scales.

What are the practices for the public to evaluate the overbalance of pandemic information?

How can digital artifacts for infodemic fact-checking be designed and implemented?

How can health officials minimize the impact of infodemic on pandemic management measures (e.g., mobility restrictions and lockdowns)?

How can complex phenomena such as 'post-truth' be theorized in the context of infodemic?

Digital tools and agency of actors

How can journalists create informative digital content via social media platforms (e.g., Twitter) to help public health authorities for informed public actions against infodemic (such as the acceptance of COVID-19 vaccines and infection prevention practices)?

How does ineffective use of social media contribute to misinformation in the context of epidemics and pandemics?

How do analyses of online content (e.g., tweets) of current and past infodemic (e.g., influenza, Ebola) can help inform public health policies?

Infodemic crisis management
How can different stockholders (e.g., governments, media, and technologists) collectively design and implement effective strategies for mitigating the misinformation crisis?

How do science and media shape public discourse in managing infodemic?

How can the negative impact of infodemic on public trust be effectively repaired?

What are the roles of information literacy on social media users' attitudes towards misinformation?

How can different media technologies effectively form public attitudes towards pandemic-related conspiracy theories? 


\begin{tabular}{ll}
\hline \multicolumn{1}{c}{ Concepts } & \multicolumn{1}{c}{ Key Questions } \\
\hline $\begin{array}{l}\text { Infodemic knowledge } \\
\text { and mental health impact }\end{array}$ & $\begin{array}{l}\text { How and why do mental health situations (e.g., anxiety, depression) of social } \\
\text { media users contribute to the consumption and sharing of infodemic, and vice } \\
\text { versa? } \\
\text { What are the relationships between health information knowledge, and literacy } \\
\text { on the acceptance and sharing misinformation in digital platforms? }\end{array}$ \\
\hline $\begin{array}{l}\text { Studying and modeling } \\
\text { the infodemic trends and } \\
\text { topics }\end{array}$ & $\begin{array}{l}\text { How do users employ social media affordances (e.g., through liking, replying, } \\
\text { and retweeting behavior) to spread infodemics? } \\
\text { What are the trends, dominant contents, and spectrums of infodemic topics that } \\
\text { affect population health? } \\
\text { How can topic modeling approaches be employed to predict and explain user } \\
\text { resistance or acceptance of misinformation by analyzing online reviews, } \\
\text { information seeking trends, and comments? }\end{array}$ \\
\hline
\end{tabular}

\section{Conclusions}

Empowered by the authorized use of pandemic data, digital technologies and platforms can facilitate better management of a pandemic. However, misuse of digital platforms including social media for generating and disseminating misinformation jeopardizes public health policies and practices such as the acceptance of COVID-19 vaccination. In this study, we specified the infodemic concepts and research topics that are worth exploring in the context of pandemics. Also, new directions are proposed for creating research impacts. Future research can examine hot infodemic topics such as social media and misinformation (i.e. studying the effective use of social media in the context of pandemics misinformation). Finally, in its 'managing the infodemic' [9] call for action, WHO proposes seven actions as the current solutions or possibilities to resolve or reduce the infodemic effects. In line with this important call, we invite medical informaticians and information systems communities to take action and contribute towards this global discourse on infodemic and public health.

\section{References}

[1] Chan AK, Nickson C, Rudolph J, Lee A, Joynt G. Social media for rapid knowledge dissemination: early experience from the COVID - 19 pandemic. Anaesthesia. 2020;75:1579-82.

[2] Bao H, Cao B, Xiong Y, Tang W. Digital media's role in the COVID-19 pandemic. JMIR mHealth and uHealth. 2020;8(9):e20156.

[3] Vosoughi S, Roy D, Aral S. The spread of true and false news online. Science. 2018;359(6380):1146-51.

[4] Zarocostas J. How to fight an infodemic. The Lancet. 2020;395(10225):676.

[5] World Health Organization. Immunizing the public against misinformation 2020, Available at: https:/www.who.int/news-room/feature-stories/detail/immunizing-the-public-against-misinformation, Accessed Jan 10, 2021.

[6] World Health Organization. Managing the COVID-19 infodemic: Promoting healthy behaviours and mitigating the harm from misinformation and disinformation 2020, Available at: https:/www.who.int/news/item/23-09-2020-managing-the-covid-19-infodemic-promoting-healthybehaviours-and-mitigating-the-harm-from-misinformation-and-disinformation, Accessed Jan 10, 2021.

[7] World Health Organization. Let's flatten the infodemic curve 2020, Available at https://www.who.int/news-room/spotlight/let-s-flatten-the-infodemic-curve, Accessed Jan 10, 2021.

[8] Van Eck NJ, Waltman L. Software survey: VOSviewer, a computer program for bibliometric mapping. scientometrics. 2010;84(2):523-38.

[9] World Health Organization. Call for Action: Managing the Infodemic 2020, Available at https://www.who.int/news/item/11-12-2020-call-for-action-managing-the-infodemic, Accessed Jan 11, 2021. 